\title{
Changes in Cardiovascular Structure and Physiology Following Polystyrene Nanoparticle Exposure
}

\author{
Zachary Lett ${ }^{\mathrm{a}}$, Shelby Skidmore ${ }^{\mathrm{a}}$, Nathan J Alves ${ }^{\mathrm{b}, \mathrm{c},{ }^{*}}$ \\ a Indiana University School of Medicine, Indianapolis, IN, United States of America \\ ${ }^{\mathrm{b}}$ Emergency Medicine Department, Indiana University School of Medicine, Indianapolis, IN, \\ United States of America \\ ${ }^{c}$ Weldon School of Biomedical Engineering, Purdue University, West Lafayette, IN, United \\ States of America \\ "Corresponding author at: Indiana University School of Medicine, 635 Barnhill Dr. Rm. 371, \\ Indianapolis, IN 46202, United States of America. E-mail address: nalves@iu.edu (N.J. Alves).
}

\section{Background}

Microplastic (MP) pollution has been a growing concern in recent decades due to the proliferation and ease of manufacturing regarding plastics products. Polystyrene (PS), being one the most abundant plastic polymers, is the subject of frequent studies due to its ubiquitous nature. Trophic transfer, inhalation, and dermal exposure are all routes by which humans may be exposed to MPs every day. There are undoubtedly more physiological consequences than we are currently aware of; however, many of these concerns are beginning to be better understood. Their distribution throughout the body dictates the potential threats to human health. The cardiovascular system is potentially the most susceptible, as it is the first medium the MPs are exposed to after entering the body. Consequently, the cardiovascular system and thrombus generation is one such area where much recent attention has been allocated.

\section{Project Methods}

Data was gathered from a variety of well-established research articles to review the relationship between PS exposure and thrombus formation in vivo. Additionally, this review looks at documented effects of MPs on platelet aggregation and discusses mechanisms through which these pathologies take place.

\section{Results}

PS plastics have varying impact on thrombus formation contingent upon both surface modifications and particle size. Aminated PS appears to be the most potent regarding the generation of thrombi in vivo. Literature on platelet aggregation is more consistent, with most studies revealing that carboxylated and aminated forms enhance aggregation while unmodified PS had insignificant effects.

\section{Conclusion/Potential Impact}

There is substantial ambiguity surrounding this field and more studies need to be conducted to reveal the full extent of pathologies caused by PS microplastics. Most studies have been done with marine life, which may not accurately reflect MPs effects in humans. Further research will 
allow us to begin developing viable solutions for those most susceptible to cardiovascular diseases. 